\title{
One-dimensional cuts through multidimensional potential-energy surfaces by tunable $\mathrm{x}$ rays
}

\author{
Sebastian Eckert, ${ }^{1,2, *}$ Vinícius Vaz da Cruz, ${ }^{3, \dagger}$ Faris Gel'mukhanov, ${ }^{3,4}$ Emelie Ertan, ${ }^{5}$ Nina Ignatova, ${ }^{3,4}$ Sergey Polyutov, ${ }^{4}$ \\ Rafael C. Couto, ${ }^{3}$ Mattis Fondell, ${ }^{2}$ Marcus Dantz, ${ }^{6}$ Brian Kennedy, ${ }^{2}$ Thorsten Schmitt, ${ }^{6}$ Annette Pietzsch, ${ }^{2}$ \\ Michael Odelius, ${ }^{5, \ddagger}$ and Alexander Föhlisch ${ }^{1,2}$ \\ ${ }^{1}$ Institut für Physik und Astronomie, Universität Potsdam, Karl-Liebknecht-Strasse 24-25, 14476 Potsdam, Germany \\ ${ }^{2}$ Institute for Methods and Instrumentation for Synchrotron Radiation Research FG-ISRR, Helmholtz-Zentrum Berlin \\ für Materialien und Energie, Albert-Einstein-Strasse 15, 12489 Berlin, Germany \\ ${ }^{3}$ Theoretical Chemistry and Biology, Royal Institute of Technology, 10691 Stockholm, Sweden \\ ${ }^{4}$ Institute of Nanotechnology, Spectroscopy, and Quantum Chemistry, Siberian Federal University, 660041 Krasnoyarsk, Russia \\ ${ }^{5}$ Department of Physics, Stockholm University, AlbaNova University Center, 10691 Stockholm, Sweden \\ ${ }^{6}$ Research Department Synchrotron Radiation and Nanotechnology, Paul Scherrer Institut, CH-5232 Villigen PSI, Switzerland
}

(Received 23 January 2018; published 18 May 2018)

\begin{abstract}
The concept of the potential-energy surface (PES) and directional reaction coordinates is the backbone of our description of chemical reaction mechanisms. Although the eigenenergies of the nuclear Hamiltonian uniquely link a PES to its spectrum, this information is in general experimentally inaccessible in large polyatomic systems. This is due to (near) degenerate rovibrational levels across the parameter space of all degrees of freedom, which effectively forms a pseudospectrum given by the centers of gravity of groups of close-lying vibrational levels. We show here that resonant inelastic $\mathrm{x}$-ray scattering (RIXS) constitutes an ideal probe for revealing one-dimensional cuts through the ground-state PES of molecular systems, even far away from the equilibrium geometry, where the independent-mode picture is broken. We strictly link the center of gravity of close-lying vibrational peaks in RIXS to a pseudospectrum which is shown to coincide with the eigenvalues of an effective one-dimensional Hamiltonian along the propagation coordinate of the core-excited wave packet. This concept, combined with directional and site selectivity of the core-excited states, allows us to experimentally extract cuts through the ground-state PES along three complementary directions for the showcase $\mathrm{H}_{2} \mathrm{O}$ molecule.
\end{abstract}

DOI: 10.1103/PhysRevA.97.053410

\section{INTRODUCTION}

Multidimensional potential-energy surfaces (PES), dynamic pathways, and reaction coordinates are powerful conceptual tools for molecular science and chemistry. Experimental reconstruction of multidimensional PES—-spanning from equilibrium to strong geometric distortion—of coupled degrees of freedom poses a challenge. Ideally, knowledge and interpretation of the full experimental vibrational spectrum are needed to solve this inverse problem [1-5]. It is almost impossible to extract such information from experimental spectra of large polyatomic systems. However, knowledge of the full multidimensional PES is redundant in many situations. Indeed, the majority of practical applications requires knowledge of the PES along directional reaction pathways, such as in imaging of folding energy landscapes by single-molecule force spectroscopy [6,7] instead of the full PES landscape along all degrees of freedom of the investigated system.

\footnotetext{
*sebeckert@uni-potsdam.de

†vvdc@kth.se

†odelius@fysik.su.se
}

Published by the American Physical Society under the terms of the Creative Commons Attribution 4.0 International license. Further distribution of this work must maintain attribution to the author(s) and the published article's title, journal citation, and DOI.
We present subnatural linewidth resonant inelastic x-ray scattering [8-11] (RIXS) as an experimental approach that delivers this directional reconstruction method of the groundstate potential-energy surface. The strong site and state sensitivity of RIXS uniquely enables probing the electronic groundstate PES in different directions using resonant $\mathrm{x}$-ray scattering through selected intermediate core-excited electronic states. The role of these intermediate states is crucial for the potential reconstruction, because it defines the selective propagation of the nuclear wave packet in state- and site-specific directions. Indeed, the core-excited-state wave packets propagate along the coordinates defined by the orientation of valleys of the selected core-excited-state PES. The projections of these directional wave packets onto vibrational states of the electronic final state, in the emission step of the RIXS process, give access to the final-state PES along the selected pathway in a wide range of geometry distortions (Fig. 1).

Quite often, vibrational states are clustered into groups of close-lying vibrational levels (typically unresolved in current RIXS spectra) [12-15]. In principle, the fine structure of each group can be resolved using RIXS of super-high resolution. However, we face here a nonintuitive situation where the knowledge of such a fine structure of overlapping vibrational states is redundant and even constitutes an obstacle in our reconstruction technique. Instead, we need only much simpler information, namely, the centers of gravity of each group, which form a pseudospectrum that is unique for each propagation direction of the wave packet on the core-excited 


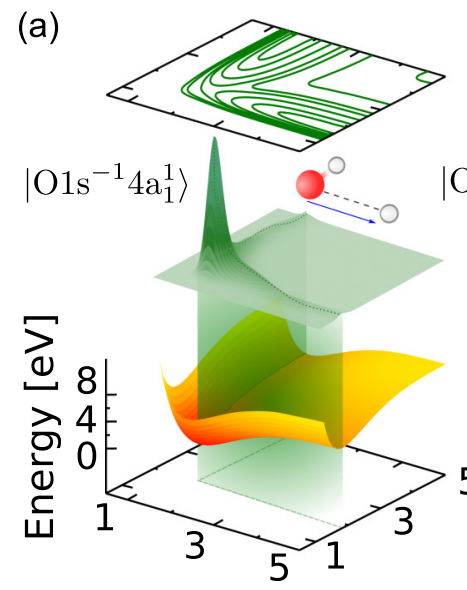

(b)
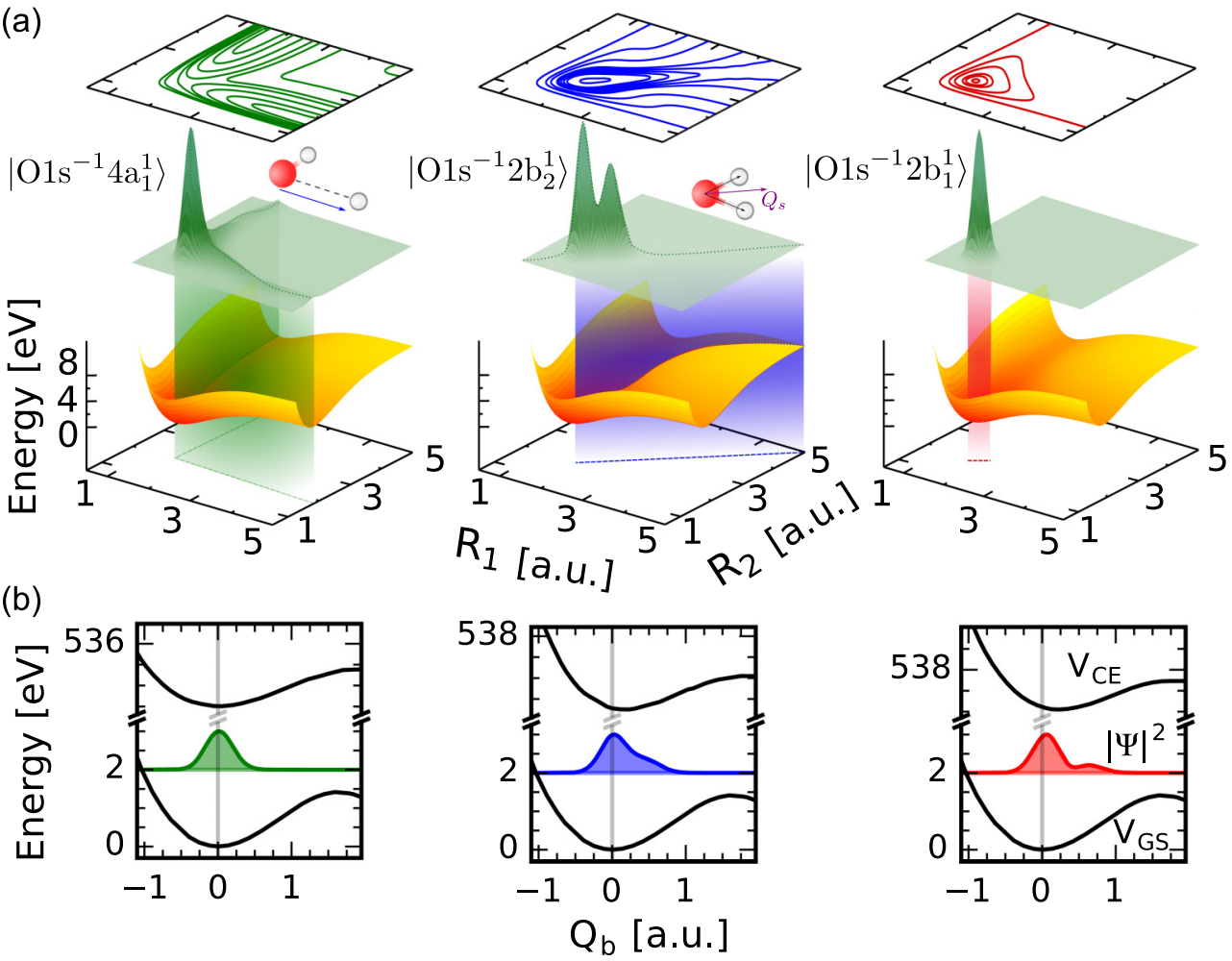

FIG. 1. Localized nuclear wave packets and 1D projections. Squared core-excited-state nuclear wave packets $|\Psi|^{2}$ of gas-phase water versus (a) $\mathrm{OH}$ bond lengths $R_{1}$ and $R_{2}$ and (b) bending normal coordinate $Q_{b} . \Psi$ exhibits a strong localization along the bonds and between the bonds for the $\left|\mathrm{O} 1 s^{-1} 4 a_{1}^{1}\right\rangle$ and $\left|\mathrm{O} 1 s^{-1} 2 b_{2}^{1}\right\rangle$ core-excited states, respectively, and is altered exclusively along $Q_{b}$ for the $\left|\mathrm{O} 1 s^{-1} 2 b_{1}^{1}\right\rangle$ excited state. The core-excited-state potential-energy surfaces $V_{\mathrm{CE}}$ drive directional wave-packet transformations. The projection $\left\langle\psi_{n_{1} n_{2}} \mid \Psi\right\rangle$ of the core-excited wave packet onto the ground state allows to reconstruct $1 \mathrm{D}$ cuts of the ground-state potential $V_{\mathrm{GS}}$ along distinct directions.

PES. We have found that this pseudospectrum allows us to extract one-dimensional cuts of the electronic ground-state potential along the propagation direction of the nuclear wave packet in the selected core-excited state.

\section{RESONANT INELASTIC X-RAY SCATTERING OF WATER MOLECULES}

To exemplify the PES reconstruction, we study the electronically elastic RIXS process in water molecules, which ends up in the electronic ground state. The O $1 s$ x-ray absorption spectrum of gas-phase water exhibits three wellseparated absorption resonances $[11,16]$ which correspond to excitations of the $\mathrm{O} 1 s$ electron into the unoccupied molecular levels $4 a_{1}, 2 b_{2}$, and $2 b_{1}$. The theoretical simulations of the core-excited-state wave-packet propagation in Fig. 1 show that the nuclear wave packet moves along different state-specific reaction coordinates on the different core-excited states within the lifetime of the O $1 s$ core hole. The wave packets, confined within the PES valleys, are localized along the $\mathrm{OH}$ bonds of the dissociative $\left|\mathrm{O} 1 s^{-1} 4 a_{1}^{1}\right\rangle$ state, between the $\mathrm{OH}$ bonds for the $\left|\mathrm{O} 1 s^{-1} 2 b_{2}^{1}\right\rangle$ potential, and exclusively along the bending coordinate for the $\left|\mathrm{O} 1 s^{-1} 2 b_{1}^{1}\right\rangle$ state [11]. Upon decay, these directional wave packets are projected onto the vibrational levels of the ground state, thus enabling reconstruction of different cuts through the PES (Fig. 1).

Here, we consider the RIXS spectra of $\mathrm{H}_{2} \mathrm{O}$ for photon energies tuned in resonance with the $\left|\mathrm{O} 1 s^{-1} 4 a_{1}^{1}\right\rangle,\left|\mathrm{O} 1 s^{-1} 2 b_{2}^{1}\right\rangle$, and $\left|\mathrm{O} 1 s^{-1} 2 b_{1}^{1}\right\rangle$ transitions. The wave packets $\Psi$ presented in Fig. 1 were used in Refs. [11] and [17] to compute the RIXS spectra at each absorption resonance and lead to good agreement with experiment. The exclusive presence of stretching excitations in the $\left|\mathrm{O} 1 s^{-1} 4 a_{1}^{1}\right\rangle$ spectrum, dominance of stretching excitations with presence of a single bending overtone in the $\left|\mathrm{O} 1 s^{-1} 2 b_{2}^{1}\right\rangle$ spectrum, and the exclusive existence of bending excitations in the $\left|\mathrm{O} 1 s^{-1} 2 b_{1}^{1}\right\rangle$ spectrum reflect the coupling of the characteristic confinement of the core-excited-state wave packets to the experimentally detected ground-state vibrational modes $[17,18]$.

\section{EXPERIMENT}

The RIXS end station of the ADRESS beam line [19] at the Swiss Light Source was utilized to acquire the experimental spectra with a resolution of $75 \mathrm{meV}$. The $\mathrm{H}_{2} \mathrm{O}(\mathrm{g})$ sample, generated by evacuation from a $\sim 10 \mathrm{ml}$ liquid sample reservoir at a temperature of $60^{\circ} \mathrm{C}$, was transferred through previously evacuated and heated steel capillaries to the interaction region under permanent evacuation. In this way a constant flow and thus continuous sample replacement was achieved. At the point of interaction with the synchrotron $\mathrm{x}$-ray beam, the sample was separated from the UHV experimental chamber by a $150-\mathrm{nm}$ thin silicon nitride membrane. The surface of the membrane was positioned under an angle of $45^{\circ}$ with respect to the incoming $\mathrm{x}$-ray beam, allowing for a transmission of both the incident as well as the emitted photons in a $90^{\circ}$ scattering geometry. 
The energy calibration of the ADRESS beam line was determined according to the absorption maximum at the $\pi^{*}$ resonance of $\mathrm{O}_{2}$ gas at $530.519 \mathrm{eV}$ to be in agreement with the data presented by Saitoh et al. [20], who reported 530.521 \pm $0.01 \mathrm{eV}$. Disagreements about this absolute energy exist in literature as pointed out by Prince et al. [21]. The nominal energies according to the beamline calibration for the spectra recorded at the different resonances are $533.509 \mathrm{eV}$ for $\left|\mathrm{O} 1 s^{-1} 4 a_{1}^{1}\right\rangle$, $535.289 \mathrm{eV}$ for $\left|\mathrm{O} 1 s^{-1} 2 b_{2}^{1}\right\rangle$, and $536.699 \mathrm{eV}$ for $\left|\mathrm{O} 1 s^{-1} 2 b_{1}^{1}\right\rangle$. From the comparison between the incident-energy-dependent experimental spectra and simulated spectra considering the detuning dependent core-excited-state dynamics we estimate the detuning from the different resonances to be $0.05 \mathrm{eV}$ for $\left|\mathrm{O} 1 s^{-1} 4 a_{1}^{1}\right\rangle,-0.025 \mathrm{eV}$ for $\left|\mathrm{O} 1 s^{-1} 2 b_{2}^{1}\right\rangle$, and $0.2 \mathrm{eV}$ for $\left|\mathrm{O} 1 s^{-1} 2 b_{1}^{1}\right\rangle$ [17].

\section{ELECTRONIC STRUCTURE METHODS AND RIXS THEORY}

To compute the potential-energy surfaces of the ground and core-excited states we employed the MOLCAS 8.0 package [22] using the scalar relativistic restricted-active-space selfconsistent field (RASSCF) method [23] followed by secondorder perturbation theory (RASPT2) method [24]. The atomic orbitals were described by an ANO-RCC [25] basis set in combination with a (2s2p1d) Rydberg basis similarly to Ref. [26]. The normal vibrational modes of the ground state were obtained at the CASPT2 $(8,9)$ level. An active space with 10 electrons consisting of 11 orbitals in RAS2 and RAS3 was used in the RASPT2 calculations (in $C_{s}$ symmetry) of the potential-energy surfaces. To reach both ground and coreexcited states, separate RASSCF calculations were performed with double or single occupation of $\mathrm{O} 1 s$ (which is frozen from the Hartree-Fock calculation) in the RAS3 subspace. RASSCF state-averaging and multistate RASPT2 were carried out for $\left|\mathrm{O} 1 s^{-1} 4 a_{1}^{1}\right\rangle$ and $\left|\mathrm{O} 1 s^{-1} 2 b_{2}^{1}\right\rangle$.

We compute the RIXS cross section assuming that the stretching and bending are uncoupled (so-called 2D+1D model $[11,17]$ using a time-dependent representation

$$
\begin{aligned}
\sigma_{f c}\left(\omega^{\prime}, \omega\right)= & \sum_{m, m_{c}^{\prime}, m_{c}}\left\langle 0 \mid m_{c}^{\prime}\right\rangle\left\langle m_{c}^{\prime} \mid m\right\rangle\left\langle m \mid m_{c}\right\rangle\left\langle m_{c} \mid 0\right\rangle \\
& \times \operatorname{Re} \int_{0}^{\infty} d t e^{i\left(\omega-\omega^{\prime}-\epsilon_{m}+\epsilon^{(0)}\right) t} c_{m_{c}^{\prime} m_{c}}(t)
\end{aligned}
$$

as the function of the energy loss $\omega-\omega^{\prime}$. Here $\omega^{\prime}$ is the frequency of the scattered photon, $\epsilon^{(0)}$ and $\epsilon_{m}$ are the total zero-point energy and the bending vibrational energy of the ground state. To find the autocorrelation function,

$$
\begin{aligned}
c_{m_{c}^{\prime} m_{c}}(t) & =\left\langle\Phi_{m_{c}^{\prime}}(0)\left|e^{-i h_{2} t}\right| \Psi_{m_{c}}\right\rangle, \\
\Psi_{m_{c}} & =\int_{0}^{\infty} d t_{1} e^{-\Gamma t_{1}} e^{i\left(\Omega-\epsilon_{m_{c}}+\Delta\right) t_{1}} \psi\left(t_{1}\right),
\end{aligned}
$$

where $\Omega=\omega-\omega_{c 0}+\epsilon^{(0)}$ is the detuning of the incoming photon frequency from the frequency $\omega_{c 0}$ of the vertical transition $0 \rightarrow c$. Here $\Delta=E_{c}\left(\mathbf{R}_{0}\right)-E_{c}\left(\mathbf{R}_{0}^{(c)}\right), \mathbf{R}_{0}$ and $\mathbf{R}_{0}^{(c)}$ are the coordinates of the potential minima of the ground and core-excited three-dimensional (3D) potentials.
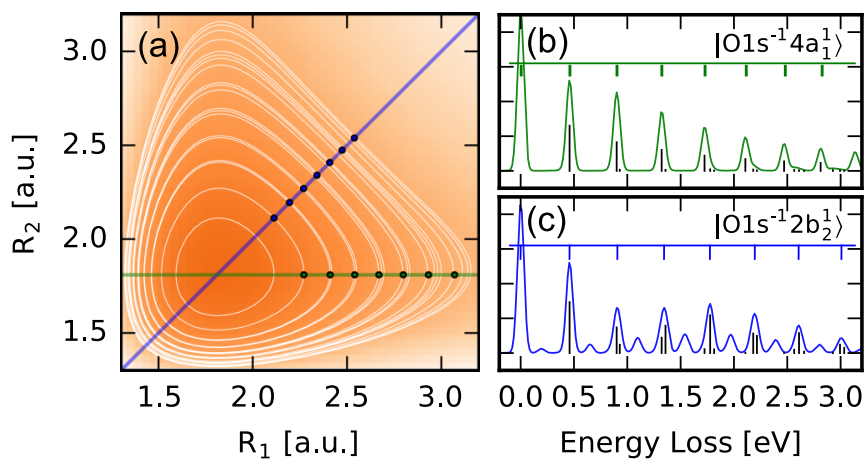

FIG. 2. From eigenstates of a multidimensional Hamiltonian to a one-dimensional pseudospectrum.(a) White curves show the crossings of the stretching isoenergetic surfaces $\epsilon_{n_{1} n_{2}}$ with the ground-state PES $V\left(R_{1}, R_{2}\right)=\epsilon_{n_{1} n_{2}}$. Circles along the coordinates $R_{1}$ (green) and $Q_{s}$ (blue) are the centers of gravity $\epsilon_{n}^{c g}$ of the $n$th group of the $\left|\mathrm{O} 1 s^{-1} 4 a_{1}^{1}\right\rangle$ and $\left|\mathrm{O} 1 s^{-1} 2 b_{2}^{1}\right\rangle$. The curves show the theoretical RIXS profile computed at the (a) $\left|\mathrm{O} 1 s^{-1} 4 a_{1}^{1}\right\rangle$ and (b) $\left|\mathrm{O} 1 s^{-1} 2 b_{2}^{1}\right\rangle$ resonances. The bars above show the pseudospectra $\epsilon_{n}=\epsilon_{n}^{\mathrm{cg}}$ which can be extracted along different coordinates from the $\left|\mathrm{O} 1 s^{-1} 4 a_{1}^{1}\right\rangle$ (b) and $\left|\mathrm{O} 1 s^{-1} 2 b_{2}^{1}\right\rangle$ (c) RIXS due to different Franck-Condon factors (shown by black vertical lines). They allow for the reconstruction of the potential along the $\mathrm{OH}$ bonds $R_{1}$ and along the symmetric stretching direction $Q_{s}$.

\section{1D CUT OF THE PES AND THE PSEUDOSPECTRUM}

Let us illustrate, based on the detected RIXS transitions, how the confined core-excited-state wave packets can be used to reconstruct one-dimensional (1D) cuts through the ground-state PES. To give insight into the physics, it is enough to consider only the coupled stretching dynamics. The two-dimensional (2D) Hamiltonian of the coupled stretching motion of the ground electronic state in its general form

$$
h_{2}=-\frac{1}{2 \mu_{1}} \frac{\partial^{2}}{\partial Q_{1}^{2}}-\frac{1}{2 \mu_{2}} \frac{\partial^{2}}{\partial Q_{2}^{2}}-\zeta \frac{\partial^{2}}{\partial Q_{1} Q_{2}}+V\left(Q_{1}, Q_{2}\right) \text {. }
$$

Here $\mu_{i}$ are the reduced masses for motion along the general coordinate $Q_{i}$. The kinetic energy operator is not diagonal due to the mass polarization term $\propto \zeta$. It is known that the eigenvalues $\epsilon_{n_{1} n_{2}}$ of the ground-state 2D Hamiltonian $h_{2}$ form groups $[15,17,18]$ according to the group number

$$
n=n_{1}+n_{2}, \quad n=0,1,2, \ldots
$$

Each $n$th group consist of $n+1$ close-lying vibrational sublevels as illustrated in Fig. 2. Such clustering of the vibrational states into groups occurs in many molecules containing $\mathrm{H}-X$ bonds (e.g., $\mathrm{H}_{2} X, \mathrm{H}_{3} X, \mathrm{H}_{4} X$ ) [11,15]. In our measurements the fine structure of each $n$th group is not resolved and each $n$th peak is instead characterized by its center of gravity,

$$
\begin{aligned}
\epsilon_{n}^{\mathrm{cg}} & =\sum_{n_{1}+n_{2}=n} P_{n_{1} n_{2}}^{(n)} \epsilon_{n_{1} n_{2}}, \\
P_{n_{1} n_{2}}^{(n)} & =\frac{\left|\left\langle\Psi \mid \psi_{n_{1} n_{2}}\right\rangle\right|^{2}}{\sum_{n_{1}+n_{2}=n}\left|\left\langle\Psi \mid \psi_{n_{1} n_{2}}\right\rangle\right|^{2}},
\end{aligned}
$$


which is sensitive to the direction of localization of the nuclear wave packet $\Psi$ in the core-excited state, as seen in Fig. 2. Indeed, the probability $P_{n_{1} n_{2}}^{(n)}$ is defined by the square of the scattering amplitude $[8,17,18]$,

$$
\begin{aligned}
F_{n_{1} n_{2}} & =-i\left\langle\psi_{n_{1} n_{2}} \mid \Psi\right\rangle, \\
\Psi & =\int_{0}^{\infty} e^{i\left(\omega-\omega_{c 0}+\epsilon_{00}+i \Gamma\right) t} \psi(t) d t, \quad \psi(t)=e^{-i h^{(c)} t} \psi_{00},
\end{aligned}
$$

which is the projection of $\Psi$ onto the vibrational eigenfunctions $\psi_{n_{1} n_{2}}$ of the ground state. Here, $\omega_{c 0}$ is the energy of the vertical transition $0 \rightarrow c, h^{(c)}$ and $\Gamma=0.08 \mathrm{eV}$ are the $2 \mathrm{D}$ Hamiltonian and the lifetime broadening of the core-excited state, respectively.

Figure 1(a) depicts the localization of the nuclear wave packet $\Psi$ in each core-excited state along the reaction path $Q_{1}$ which is directed into a valley of the corresponding PES. For the $\left|\mathrm{O} 1 s^{-1} 4 a_{1}^{1}\right\rangle$ dissociative state, the coordinate $Q_{1}$ is oriented along one $\mathrm{OH}$ bond $\left(Q_{1}=R_{1}\right.$ or $\left.R_{2}\right)$ and for the bound $\left|\mathrm{O} 1 s^{-1} 2 b_{2}^{1}\right\rangle$ state, it is located between the $\mathrm{OH}$ bonds (i.e., along the symmetric stretching coordinate $Q_{1}=Q_{s}$ ). The wave packet $\Psi$ evolving along $Q_{1}$ is narrow in the direction $Q_{2}$ orthogonal to $Q_{1}$. We describe the strong confinement of $\Psi$ in the $Q_{2}$ direction by the sharp function $\Delta\left(Q_{2}\right)$ (normalized to one) using the following factorization:

$$
\Psi\left(Q_{1}, Q_{2}\right)=\phi\left(Q_{1}\right) \Delta\left(Q_{2}-Q_{2 \mathrm{e}}\right),
$$

where $\Delta\left(Q_{2}-Q_{2 \mathrm{e}}\right)$ has its maximum at $Q_{2}=Q_{2 \mathrm{e}}$.

To connect the projection of this narrow wave packet (7) onto the ground-state PES with the potential $V\left(Q_{1}, Q_{2 \mathrm{e}}\right)$ along the confinement coordinate $Q_{1}$ we study the relation between the center of gravity $\epsilon_{n}^{\mathrm{cg}}(5)$ and the $n$th group to the eigenvalue $\epsilon_{n}$ of the 1D Hamiltonian $h_{1}$ along $Q_{1}$ :

$$
\begin{aligned}
h_{1} \psi_{n}\left(Q_{1}\right) & =\epsilon_{n} \psi_{n}\left(Q_{1}\right), \\
h_{1} & \equiv\left\langle\Delta\left|h_{2}\right| \Delta\right\rangle=-\frac{1}{2 \mu_{1}} \frac{\partial^{2}}{\partial Q_{1}^{2}}+V\left(Q_{1}, Q_{2 \mathrm{e}}\right)+C .
\end{aligned}
$$

The following approximation $V\left(Q_{1}, Q_{2}\right) \approx V\left(Q_{1}, Q_{2 \mathrm{e}}\right)+$ $\kappa\left(Q_{2}-Q_{2 \mathrm{e}}\right)^{2} / 2$ is valid because $\left(Q_{2}-Q_{2 \mathrm{e}}\right)$ is small. The constant offset $C=\left\langle\Delta\left|\kappa\left(Q_{2}-Q_{2 \mathrm{e}}\right)^{2} / 2+T\left(Q_{2}\right)\right| \Delta\right\rangle$ of the 1D Hamiltonian $h_{1}$ is not important because we define the potential with respect to the bottom of the well. Clearly, the spectrum of the 1D Hamiltonian $\epsilon_{n}$ depends on the statesensitive propagation direction of the wave packet $\phi\left(Q_{1}\right)$, in full agreement with experiment and simulations. Let us write the normalized wave packet $\phi\left(Q_{1}\right)$ in terms of eigenfunctions $\psi_{n}\left(Q_{1}\right)$ :

$$
\begin{aligned}
|\phi\rangle= & \left|\psi_{n}\right\rangle\left\langle\psi_{n} \mid \phi\right\rangle+\sum_{m(\neq n)}\left|\psi_{m}\right\rangle\left\langle\psi_{m} \mid \phi\right\rangle, \\
\left\langle\Psi \mid \psi_{n_{1} n_{2}}\right\rangle= & \left\langle\phi \Delta \mid \psi_{n_{1} n_{2}}\right\rangle=\left\langle\phi \mid \psi_{n}\right\rangle\left\langle\psi_{n} \Delta \mid \psi_{n_{1} n_{2}}\right\rangle \\
& +\sum_{m(\neq n)}\left\langle\phi \mid \psi_{m}\right\rangle\left\langle\psi_{m} \Delta \mid \psi_{n_{1} n_{2}}\right\rangle .
\end{aligned}
$$

One should notice that the overlap between $\left|\psi_{m} \Delta\right\rangle$ and $\left|\psi_{n_{1} n_{2}}\right\rangle$ which belong to different groups is negligible due to the small spacing $\delta \epsilon_{n_{1} n_{2}}$ between the levels inside of the same group $\left(n_{1}+n_{2}=n\right)$ in comparison with the spacing $\epsilon_{n}-\epsilon_{m}$ between the levels from different groups $(n \neq m)[11,17]$ (see Appendix):

$$
\left\langle\psi_{m} \Delta \mid \psi_{n_{1} n_{2}}\right\rangle \approx 0, \quad n_{1}+n_{2}=n \neq m .
$$

This together with the identity $\left\langle\psi_{m} \mid \psi_{n}\right\rangle=0$ allows us to write Eq. (5) as follows:

$$
\begin{aligned}
P_{n_{1} n_{2}}^{(n)} & =\frac{\left|\left\langle\psi_{n} \Delta \mid \psi_{n_{1} n_{2}}\right\rangle\right|^{2}}{\sum_{n_{1}+n_{2}=n}\left\langle\psi_{n} \Delta \mid \psi_{n_{1} n_{2}}\right\rangle\left\langle\psi_{n_{1} n_{2}} \mid \psi_{n} \Delta\right\rangle} \\
& =\left|\left\langle\psi_{n} \Delta \mid \psi_{n_{1} n_{2}}\right\rangle\right|^{2} .
\end{aligned}
$$

Thus Eqs. (5) and (11) bring about a central result of our article:

$$
\begin{aligned}
\epsilon_{n}^{\mathrm{cg}} & =\sum_{n_{1}+n_{2}=n}\left\langle\psi_{n} \Delta \mid \psi_{n_{1} n_{2}}\right\rangle \epsilon_{n_{1} n_{2}}\left\langle\psi_{n_{1} n_{2}} \mid \psi_{n} \Delta\right\rangle \\
& =\left\langle\psi_{n} \Delta\left|h_{2}\right| \psi_{n} \Delta\right\rangle=\left\langle\psi_{n}\left|h_{1}\right| \psi_{n}\right\rangle=\epsilon_{n} .
\end{aligned}
$$

It is striking that the center of gravity of the $n$th RIXS peak is nothing else than the eigenvalue $\epsilon_{n}$ of a 1D Hamiltonian $h_{1}=\left\langle\Delta\left|h_{2}\right| \Delta\right\rangle$ along the $Q_{1}$ coordinate. Hence, the set of centers of gravity of the peaks constitutes a pseudospectrum from which we can reconstruct a $1 \mathrm{D}$ potential along $Q_{1}$ of the multidimensional PES, either by employing an analytical model or a model-free numerical procedure. The pseudospectra, related to the selected cuts, are shown in Fig. 2 for the theoretical RIXS spectra computed at the $\left|\mathrm{O} 1 s^{-1} 4 a_{1}^{1}\right\rangle$ and $\left|\mathrm{O} 1 s^{-1} 2 b_{2}^{1}\right\rangle$ resonances. Figure 2 shows the sensitivity of the pseudospectrum on the direction of the 1D cut.

\section{EXPERIMENTALLY RECONSTRUCTED POTENTIALS}

Now, we are in position to validate our concept by extracting the potential cuts from the experimental RIXS spectra of $\mathrm{H}_{2} \mathrm{O}$ (Fig. 3). To reconstruct the 1D cuts we have fitted the experimental pseudospectrum $\epsilon_{v}$ to the spectrum

$$
\epsilon_{v}=\omega_{e}\left(v+\frac{1}{2}\right)-\omega_{e} x_{e}\left(v+\frac{1}{2}\right)^{2}
$$

of the Morse potential [27]

$$
V_{M}\left(Q-Q_{e}\right)=D\left(1-e^{-\alpha\left(Q-Q_{e}\right)}\right)^{2}
$$

by varying the parameters $\omega_{e}$ and $\omega_{e} x_{e}$. Here the dissociation energy $D=\omega_{e}^{2} /\left(4 \omega_{e} x_{e}\right)$ and $\alpha=\sqrt{2 \mu \omega_{e} x_{e}}$ relate to the fitted parameters.

We start the PES reconstructions, from experimental RIXS data, by studying the scattering through the dissociative $\left|\mathrm{O} 1 s^{-1} 4 a_{1}^{1}\right\rangle$ core-excited state. The molecule undergoes an ultrafast dissociation along the $\mathrm{OH}$ bonds (along $R_{1}$ and $R_{2}$, see Fig. 1), which occurs during the $\mathrm{O} 1 s$ core-hole lifetime of $1 / 2 \Gamma=4.1 \mathrm{fs}$. This gives rise to a long stretching vibrational progression, which allows to reconstruct the $1 \mathrm{D}$ potential in the direction of the $\mathrm{OH}$ bond $Q_{1}=R_{1}$ in wide range of $R_{1}$. The RIXS spectrum at the $\left|\mathrm{O} 1 s^{-1} 4 a_{1}^{1}\right\rangle$ resonance [see Fig. 3(a)] exhibits exclusively stretching excitations. Naturally, this progression is not contaminated by the vibrations of the fragment of dissociation $\mathrm{OH}$, as the signal of this species forms the so-called atomic peak [8,28], which is well separated from the main progression in RIXS. The fit of the pseudospectrum $\epsilon_{n}=\epsilon_{n}^{\mathrm{cg}}$ from the vibrationally resolved RIXS spectrum by the eigenstates of the Morse potential $V_{M}\left(Q-Q_{e}\right)$ with $R_{e}=1.81$ a.u. provides us the vibrational 

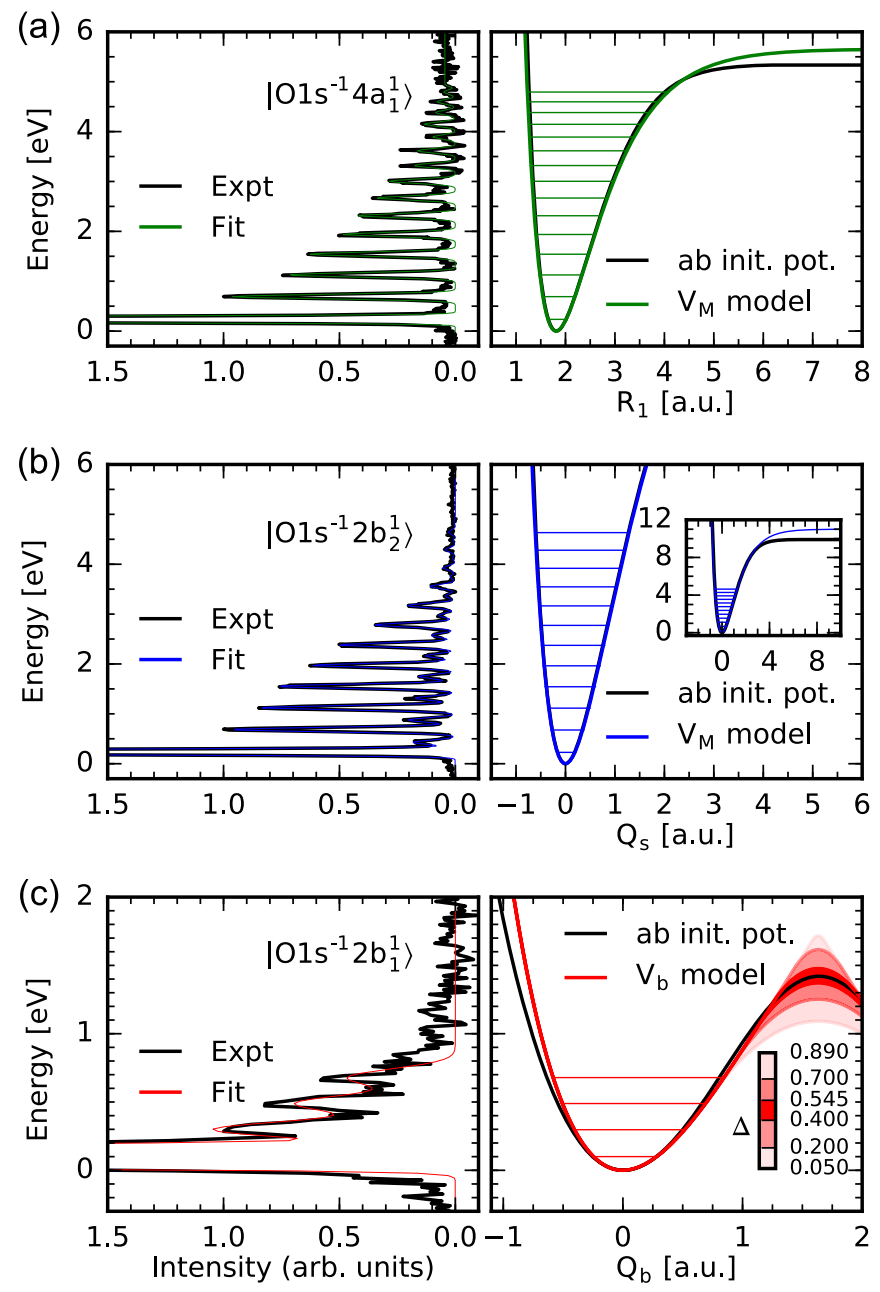

FIG. 3. Reconstruction of the $1 \mathrm{D}$ cut of the PES along the $R_{1}, Q_{s}$ (between the $\mathrm{OH}$ bonds) and $Q_{b}$ coordinate from the experimental $\left|\mathrm{O} 1 s^{-1} 4 a_{1}^{1}\right\rangle,\left|\mathrm{O} 1 s^{-1} 2 b_{2}^{1}\right\rangle$, and $\left|\mathrm{O} 1 s^{-1} 2 b_{1}^{1}\right\rangle$ RIXS spectra due to strong wave-packet confinement. (Left) The centers of gravity of each vibrational peak form the pseudospectrum (colored horizontal lines) of the ground-state $1 \mathrm{D}$ potential along the coordinates $R_{1}, Q_{s}$, and $Q_{b}$ selected by the nuclear wave packets highly confined in the valleys of the core-excited states. The fit of the pseudospectrum to the Morse potential $V_{M}$ [27] defines its parameters $\omega_{e}=(475.4 \pm 1.1) \mathrm{meV}$ and $\omega_{e} x_{e}=(9.99 \pm 0.14) \mathrm{meV}$ for $\left|\mathrm{O} 1 s^{-1} 4 a_{1}^{1}\right\rangle$ and $\omega_{e}=(457.65 \pm$ $0.70) \mathrm{meV}$ and $\omega_{e} x_{e}=(4.76 \pm 0.13) \mathrm{meV}$ for $\left|\mathrm{O} 1 s^{-1} 2 b_{2}^{1}\right\rangle$. The fit of the bending potential eigenstates yields $\omega_{e}=(201 \pm 2) \mathrm{meV}$ and $\omega_{e} x_{e}=(1.9 \pm 0.4) \mathrm{meV}$ for the model $V_{b}$. Used effective masses are given in the text. (Right) The reconstructed potentials (colored) are in good agreement with the ab initio potentials (black) in the energy range of available vibrational states.

frequency $\omega_{e}=(475.4 \pm 1.1) \mathrm{meV}$ and the anharmonicity constant $\omega_{e} x_{e}=(9.99 \pm 0.14) \mathrm{meV}$. Together with the value of the reduced mass $\mu_{1}=m_{\mathrm{H}} m_{\mathrm{O}} /\left(m_{\mathrm{H}}+m_{\mathrm{O}}\right)=0.94$ a.m.u., this yields the following parameters of the Morse potential: $\alpha=1.122 \pm 0.008$ a.u., $D=5.66 \pm 0.09 \mathrm{eV}$. In spite of our experimental data not providing $\epsilon_{n}$ in the vicinity of the dissociation limit, the obtained dissociation energy is rather close to the single $\mathrm{O}-\mathrm{H}$ bond dissociation energy of $\sim 5.6 \mathrm{eV}$ in a gas-phase water molecule [29]. The reconstructed potential is compared to the calculated $1 \mathrm{D}$ potential along the $\mathrm{OH}$ bond in Fig. 3(a). Both potentials are almost identical in the range where vibrational excitations are detected.

Let us tune the photon energy in resonance with the $\left|\mathrm{O} 1 s^{-1} 2 b_{2}^{1}\right\rangle$ core-excited state, where the wave packet $\Psi$ is confined within the narrow potential well (Fig. 1) between the bonds. Contrary to the previous case, we are able now to get a cut of the ground-state PES along symmetric bond elongations $Q_{1}=Q_{s}$ (see Fig. 1). The stretching pseudospectrum $\epsilon_{n}=$ $\epsilon_{n}^{\mathrm{cg}}$ extracted from the $\left|\mathrm{O} 1 s^{-1} 2 b_{2}^{1}\right\rangle$ RIXS spectrum fitted by the eigenstates of the Morse potential $V_{M}$ gives values of the vibrational frequency $\omega_{e}=(457.65 \pm 0.70) \mathrm{meV}$ and the anharmonicity constant $\omega_{e} x_{e}=(4.76 \pm 0.13) \mathrm{meV}$. Taking the reduced mass for the symmetric stretching bond elongation $\mu_{s}=1.044$ a.m.u. into account we get $\alpha=0.82 \pm 0.02$ a.u., $D=11.0 \pm 0.3 \mathrm{eV}$ as parameters of the Morse potential $V_{M}$. The deviation of the obtained value of $D$ from the thermochemical dissociation energy of $9.5 \mathrm{eV}$ [31] is caused by the fact that the vibrational progression is not long enough for the studied state to accurately predict the dissociation limit. Comparing the reconstructed potential to the $a b$ initio cut along $\mathrm{Q}_{s}$ in Fig. 3(b), it becomes obvious that there is a nearly perfect overlap between experiment and the theoretically predicted potential in the probed range.

Lastly, we consider the scattering through the $\left|\mathrm{O} 1 s^{-1} 2 b_{1}^{1}\right\rangle$ resonance [see Fig. 3(c)]. This spectrum exhibits only bending excitations because the $2 \mathrm{D}$ stretching potentials of the $\left|\mathrm{O} 1 s^{-1} 2 b_{1}^{1}\right\rangle$ and ground states are the same [11]. Thus, the nuclear wave packet $\Psi$ in the $\left|\mathrm{O} 1 s^{-1} 2 b_{1}^{1}\right\rangle$ core-excited state propagates only along the bending coordinate $Q_{b}$ [Fig. 1(b)]. The projection of $\Psi$ trapped in the valley of the bending potential onto the ground state allows us to recover the 1D cut of the ground-state PES along $Q_{b}$. The bending potential of $\mathrm{H}_{2} \mathrm{O}$ has two minima $\left(Q_{e 1}=0\right.$ and $Q_{e 2}=3.891$ a.u.), symmetric around the angle $\theta=\angle(H O H)=180^{\circ}$, which corresponds to the top of the barrier at $Q_{e}=1.632$ a.u. Therefore, we use a piecewise defined potential function $V_{b}$ formed by two nonoverlapping Morse potentials $V_{M}(Q)$ and $V_{M}\left[-\left(Q-Q_{e 2}\right)\right]$ (14) connected by a parabolic barrier $U\left(Q-Q_{e}\right)=U_{\max }-\eta\left(Q-Q_{e}\right)^{2}$ :

$$
V_{b}(Q)=\left\{\begin{array}{l}
V(Q), Q \leqslant-\Delta+Q_{e} \\
U\left(Q-Q_{e}\right),-\Delta+Q_{e} \leqslant Q \leqslant \Delta+Q_{e}, \\
V\left(-\left(Q-2 Q_{e}\right)\right), Q \geqslant \Delta+Q_{e} .
\end{array}\right.
$$

The morse potentials and $U\left(Q-Q_{e}\right)$ are matched at the points $Q_{e} \pm \Delta$, where $\Delta$ is a fitting parameter. The parameters $\omega_{e}=(201 \pm 2) \mathrm{meV}, \omega_{e} x_{e}=(1.9 \pm 0.4) \mathrm{meV}$, and $\Delta>Q_{e}$ were determined by a fit to the spectrum of the potential $V_{b}$ to the experimental data, with the mass of the bending mode $\mu=\mu_{b}=1.083$ a.m.u. The reconstructed potential along the bending coordinate $Q_{b}$ is compared to the ab initio simulation in Fig. 3(c). The limited number of vibrational states in the experimental spectrum does not allow to define precisely the height $U_{\max }$ of the barrier $U\left(Q-Q_{e}\right)$. However, the least-squares method employed yields a confidence interval of $1.09 \mathrm{eV}<U_{\max }<1.70 \mathrm{eV}$ [see Fig. 3(c)] for the linearity barrier. This confidence interval is consistent with the $a b$ initio values given by our RASPT2 calculations $\left(U_{\max }=1.42 \mathrm{eV}\right)$ and the coupled cluster value from Ref. [30] $\left(U_{\max }=1.37 \mathrm{eV}\right)$. 


\section{CONCLUSIONS}

A rigorous calculation of multidimensional potentialenergy surfaces is a formidable computational task, especially far away from equilibrium where accurate, but computationally costly, multiconfigurational techniques are required. Hence, there is a vital need to reconstruct the potentials directly from the experiment. In this study we validated a method to experimentally extract one-dimensional cuts through the multidimensional ground-state PES of molecular systems using vibrationally resolved RIXS spectra of the water molecule. We utilized the diverse nature of three different $\mathrm{O} 1 s$ core-excited PES to prepare highly directional nuclear wave packets which were projected onto the ground state in the RIXS process. The physical reason for the reduction of dimensionality, and for the related selection of the directions of the one-dimensional cut, is the high confinement of the wave packet along the valleys of the PES oriented differently for each core-excited state. The quantum-mechanical justification for the extractions of the selected one-dimensional potential cuts was given by relating the pseudospectrum, defined as the centers of gravity of clustered vibrational peaks measured in RIXS spectra, to the eigenstates of the one-dimensional Hamiltonian along the direction of propagation of the core-excited wave packet.

Core-excited states of different elemental sites $(\mathrm{C}, \mathrm{O}, \mathrm{N}$, etc.) have distinct PESs. These manifolds of intermediate-state PES topologies significantly enlarge the number of directions for 1D cuts of PES which are accessible through RIXS. As shown by many experimental studies of polyatomic systems $[5,13,32,33]$, only a few vibrational modes related to the core-excited atom are active. This state- and site-specific selection of only a few excitation-center-related vibrational modes, together with direct access to high vibrational states in a single shot, makes RIXS a powerful method, yielding access to cuts through PESs in molecules over highly distorted geometries, which complements other vibrational probes.

\section{ACKNOWLEDGMENTS}

This work was supported by grants from the Swedish National Infrastructure for Computing (SNIC), SNIC 2015/169 and SNIC 023/07-18, and the Swedish Research Council Grant No. 2015-03781 (VR). F.G., N.I., and S.P. acknowledge support from Russian Scientific Foundation (Project No. 1612-10109); V.C.C. and R.C.C. acknowledge the Conselho Nacional de Desenvolvimento Científico e Tecnológico (CNPqBrazil). R.C.C. acknowledges the Carl Tryggers Foundation (CTS 15:266) and the Knut and Alice Wallenberg Foundation (Grant No. KAW-2013.0020) for financial support. A.F. and M.O. acknowledge funding from the Helmholtz Virtual Institute VI419, "Dynamic Pathways in Multidimensional Landscapes." S.E. and A.F. acknowledge funding from the ERC-ADG-2014 - Advanced Investigator Grant No. 669531 EDAX under the Horizon 2020 EU Framework Programme for Research and Innovation.

APPENDIX: PROOF OF EQ. (10)

Let us show that

$$
\left\langle\psi_{m} \Delta \mid \psi_{n_{1} n_{2}}\right\rangle \approx 0, \quad m \neq n=n_{1}+n_{2}
$$

taking into account that the spacing $\delta \epsilon_{n_{1} n_{2}}$ between levels $\epsilon_{n_{1} n_{2}}$ within the $n=n_{1}+n_{2}$ manifold is much smaller than the spacing $\delta \epsilon_{n}$ between the eigenvalues $\epsilon_{n}$ of the 1D Hamiltonian $h_{1}$,

$$
\delta \epsilon_{n_{1} n_{2}} \ll\left|\epsilon_{m}-\epsilon_{n}\right|, \quad n \neq m .
$$

Here

$$
\begin{aligned}
h_{2}\left|\psi_{n_{1} n_{2}}\right\rangle & =\epsilon_{n_{1} n_{2}}\left|\psi_{n_{1} n_{2}}\right\rangle, \\
h_{1} \psi_{m} & =\epsilon_{m} \psi_{m} .
\end{aligned}
$$

Let us expand the wave packet $\left|\psi_{m}\left(Q_{1}\right) \Delta\left(Q_{2}\right)\right\rangle$ in the series over full set of eigenfunctions $\left|\psi_{n_{1} n_{2}}\right\rangle$ of a strict Hamiltonian $h_{2}=h_{1}+\delta h$ :

$$
\begin{aligned}
\left|\psi_{m}\left(Q_{1}\right) \Delta\left(Q_{2}\right)\right\rangle & =\sum_{n_{1} n_{2}} c_{n_{1} n_{2}}\left|\psi_{n_{1} n_{2}}\right\rangle, \\
\left|\psi_{m}\left(Q_{1}\right) \Delta\left(Q_{2}\right)\right\rangle & =\left|\psi_{m}\left(Q_{1}\right)\right\rangle\left|\Delta\left(Q_{2}\right)\right\rangle .
\end{aligned}
$$

To find the expansion coefficients $c_{n_{1} n_{2}}$, let us use the identity $h_{2}\left|\psi_{m} \Delta\right\rangle=\left(h_{1}+\delta h\right)\left|\psi_{m} \Delta\right\rangle$, Eq. (A3), and the expansion (A4):

$$
\begin{aligned}
\left(h_{1}+\delta h\right)\left|\psi_{m} \Delta\right\rangle & =\left(\epsilon_{m}+\delta h\right)\left|\psi_{m} \Delta\right\rangle \\
& =\left(\epsilon_{m}+\delta h\right) \sum_{k_{1} k_{2}} c_{k_{1} k_{2}}\left|\psi_{k_{1} k_{2}}\right\rangle \\
& =h_{2} \sum_{k_{1} k_{2}} c_{k_{1} k_{2}}\left|\psi_{k_{1} k_{2}}\right\rangle \\
& =\sum_{k_{1} k_{2}} c_{k_{1} k_{2}} \epsilon_{k_{1} k_{2}}\left|\psi_{k_{1} k_{2}}\right\rangle,
\end{aligned}
$$

where $\delta h=h_{2}-h_{1}$ is the deviation of the strict Hamiltonian from $h_{1}$. Multiplying this equation (from left) by $\left\langle\psi_{n_{1} n_{2}}\right|$ we get the following eigenvalue problem:

$$
\left(\epsilon_{m}-\epsilon_{n_{1} n_{2}}\right) c_{n_{1} n_{2}}+\sum_{k_{1} k_{2}}\left\langle\psi_{n_{1} n_{2}}|\delta h| \psi_{k_{1} k_{2}}\right\rangle c_{k_{1} k_{2}}=0 .
$$

This equation results in the following expression for the expansion coefficients:

$$
\begin{aligned}
c_{n_{1} n_{2}} & =\frac{A_{n_{1} n_{2}}^{(m)}}{\epsilon_{m}-\epsilon_{n_{1} n_{2}}}, \\
A_{n_{1} n_{2}}^{(m)} & \equiv-\sum_{k_{1} k_{2}}\left\langle\psi_{n_{1} n_{2}}|\delta h| \psi_{k_{1} k_{2}}\right\rangle c_{k_{1} k_{2}} .
\end{aligned}
$$

Let us write down these coefficients for two distinct cases:

$$
\begin{gathered}
c_{n_{1} n_{2}}=\frac{A_{n_{1} n_{2}}^{(m)}}{\epsilon_{m}-\epsilon_{n_{1} n_{2}}}, \quad n_{1}+n_{2} \neq m, \\
c_{m_{1} m_{2}}=\frac{A_{m_{1} m_{2}}^{(m)}}{\epsilon_{m}-\epsilon_{m_{1} m_{2}}}, \quad m_{1}+m_{2}=m .
\end{gathered}
$$

Now one can write the ratio of our interest:

$$
\begin{aligned}
\frac{c_{n_{1} n_{2}}}{c_{m_{1} m_{2}}} & =\frac{A_{n_{1} n_{2}}^{(m)}}{A_{m_{1} m_{2}}^{(m)}}\left(\frac{\epsilon_{m}-\epsilon_{m_{1} m_{2}}}{\epsilon_{m}-\epsilon_{n_{1} n_{2}}}\right) \\
& \approx \frac{A_{n_{1} n_{2}}^{(m)}}{A_{m_{1} m_{2}}^{(m)}}\left(\frac{\delta \epsilon_{m_{1} m_{2}}}{\epsilon_{m}-\epsilon_{n}}\right) .
\end{aligned}
$$


Apparently, the sum $A_{n_{1} n_{2}}^{(m)}$ [see Eq. (A7)] has the same order of magnitude for different pairs $\left(n_{1} n_{2}\right)$ :

$$
A_{n_{1} n_{2}}^{(m)} \approx A_{m_{1} m_{2}}^{(m)} .
$$

Thus we get the final result, taking into account the property (A2) of our spectrum:

$$
\left|\frac{c_{n_{1} n_{2}}}{c_{m_{1} m_{2}}}\right| \approx\left|\frac{\delta \epsilon_{m_{1} m_{2}}}{\epsilon_{m}-\epsilon_{n}}\right| \ll 1 .
$$

This means that the main contribution in the expansion (A4) of $\left|\psi_{m} \Delta\right\rangle$ gives the eigenfunction of the $m$ th group:

$$
\begin{aligned}
\left|\psi_{m} \Delta\right\rangle & \approx \sum_{m_{1}^{\prime}+m_{2}^{\prime}=m} c_{m_{1}^{\prime} m_{2}^{\prime}}\left|\psi_{m_{1}^{\prime} m_{2}^{\prime}}\right\rangle \\
& =\sum_{m_{1}^{\prime}+m_{2}^{\prime}=m}\left|\psi_{m_{1}^{\prime} m_{2}^{\prime}}\right\rangle\left\langle\psi_{m_{1}^{\prime} m_{2}^{\prime}} \mid \psi_{m} \Delta\right\rangle, \\
\left\langle\psi_{n_{1} n_{2}} \mid \psi_{m} \Delta\right\rangle & \approx 0, \quad n_{1}+n_{2} \neq m .
\end{aligned}
$$

[1] P. Maksyutenko, J. S. Muenter, N. F. Zobov, S. V. Shirin, O. L. Polyansky, T. R. Rizzo, and O. V. Boyarkin, J. Chem. Phys. 126, 241101 (2007).

[2] M. Grechko, O. V. Boyarkin, T. R. Rizzo, P. Maksyutenko, N. F. Zobov, S. V. Shirin, L. Lodi, J. Tennyson, A. G. Császár, and O. L. Polyansky, J. Chem. Phys. 131, 221105 (2009).

[3] A. G. Császár, E. Mátys, T. Szidarovszky, L. Lodi, N. F. Zobov, S. V. Shirin, O. L. Polyansky, and J. Tennyson, J. Quant. Spectrosc. Radiat. Transf. 111, 1043 (2010).

[4] C. Miron, C. Nicolas, O. Travnikova, P. Morin, Y.-P. Sun, F. Gel'mukhanov, N. Kosugi, and V. Kimberg, Nat. Phys. 8, 135 (2012).

[5] S. Schreck, A. Pietzsch, B. Kennedy, C. Såthe, P. S. Miedema, S. Techert, V. N. Strocov, T. Schmitt, F. Hennies, J.-E. Rubensson, and A. Föhlisch, Sci. Rep. 7, 20054 (2016).

[6] M. T. Woodside, P. C. Anthony, W. M. Behnke-Parks, K. Larizadeh, D. Herschlag, and S. M. Block, Science 314, 1001 (2006).

[7] A. P. Manuela, J. Lamberta, and M. T. Woodside, Proc. Natl. Acad. Sci. USA 112, 7183 (2015).

[8] F. Gel'mukhanov and H. Ågren, Phys. Rep. 312, 87 (1999).

[9] F. Hennies, A. Pietzsch, M. Berglund, A. Föhlisch, T. Schmitt, V. Strocov, H. O. Karlsson, J. Andersson, and J.-E. Rubensson, Phys. Rev. Lett. 104, 193002 (2010).

[10] A. Pietzsch, Y.-P. Sun, F. Hennies, Z. Rinkevicius, H. O. Karlsson, T. Schmitt, V. N. Strocov, J. Andersson, B. Kennedy, J. Schlappa, A. Föhlisch, J.-E. Rubensson, and F. Gel'mukhanov, Phys. Rev. Lett. 106, 153004 (2011).

[11] R. C. Couto, V. Vaz da Cruz, E. Ertan, S. Eckert, M. Fondell, M. Dantz, B. Kennedy, T. Schmitt, A. Pietzsch, F. F. Guimarães, H. Ågren, F. Gel'mukhanov, M. Odelius, V. Kimberg, and A. Föhlisch, Nat. Commun. 8, 14165 (2017).

[12] D. S. Perry, J. Mol. Spectrosc. 257, 1 (2009).

[13] Y.-P. Sun, F. Hennies, A. Pietzsch, B. Kennedy, T. Schmitt, V. N. Strocov, J. Andersson, M. Berglund, J.-E. Rubensson, K. Aidas, F. Gel'mukhanov, M. Odelius, and A. Föhlisch, Phys. Rev. B 84, 132202 (2011).

[14] E. L. Sibert III, Mol. Phys. 111, 2093 (2013).

[15] P. Jensen, WIREs Comput. Mol. Sci. 2, 494 (2012).

[16] S. Myneni, Y. Luo, L. A. Näslund, M. Cavalleri, L. Ojamäe, H. Ogasawara, A. Pelmenschikov, Ph. Wernet, P. Väterlein, C. Heske, Z. Hussain, L. G. M. Petterson, and A. Nilsson, J. Phys.: Condens. Matter 14, L213 (2002).
[17] V. Vaz da Cruz, E. Ertan, R. C. Couto, S. Eckert, M. Fondell, M. Dantz, B. Kennedy, T. Schmitt, A. Pietzsch, F. F. Guimarães, H. Ågren, F. Gel'mukhanov, M. Odelius, A. Föhlisch, and V. Kimberg, Phys. Chem. Chem. Phys. 19, 19573 (2017).

[18] N. Ignatova, V. Vaz da Cruz, R. C. Couto, E. Ertan, A. Zimin, F. F. Guimarães, S. Polyutov, H. Ågren, V. Kimberg, M. Odelius, and F. Gel'mukhanov, Sci. Rep. 7, 43891 (2017).

[19] V. N. Strocov, J. Synchrotron Radiat. 17, 631 (2010).

[20] Y. Saitoh, H. Kimura, Y. Suzuki, T. Nakatani, T. Matsushita, T. Muro, T. Miyahara, M. Fujisawa, K. Soda, S. Ueda, H. Harada, M. Kotsugi, A. Sekiyama, and S. Suga, Rev. Sci. Instrum. 71, 3254 (2000).

[21] K. C. Prince, R. Richter, M. de Simone, M. Alagia, and M. Coreno, J. Phys. Chem. A 107, 1955 (2003).

[22] F. Aquilante, J. Autschbach, R. K. Carlson, L. F. Chibotaru, M. G. Delcey, L. De Vico, I. F. Galván, N. Ferré, L. M. Frutos, L. Gagliardi et al., J. Comput. Chem. 37, 506 (2016).

[23] J. Olsen, P. Jørgensen, and J. Simons, Chem. Phys. Lett. 169, 463 (1990).

[24] P.-Å. Malmqvist, A. Rendell, and B. O. Roos, J. Phys. Chem. 94, 5477 (1990).

[25] B. O. Roos, R. Lindh, P.-Å. Malmqvist, V. Veryazov, and P. O. Widmark, J. Phys. Chem. A 108, 2851 (2004).

[26] L. Weinhardt, E. Ertan, M. Iannuzzi, M. Weigand, O. Fuchs, M. Bär, M. Blum, J. D. Denlinger, W. Yang, E. Umbach, M. Odelius and C. Heske, Phys. Chem. Chem. Phys. 17, 27145 (2015).

[27] P. M. Morse, Phys. Rev. 34, 57 (1929).

[28] X.-J. Liu, Q. Miao, F. Gel'mukhanov, M. Patanen, O. Travnikova, C. Nicolas, H. Ågren, K. Ueda, and C. Miron, Nat. Photon. 9, 120 (2015).

[29] V. O. Boyarkin, Chem. Phys. Lett. 568-569, 14 (2013).

[30] A. G. Császár, W. D. Allen, H. F. Schaefer III, J. Chem. Phys. 108, 9751 (1998).

[31] B. de B. Darwent, Bond Dissociation Energies in Simple Molecules (US National Bureau of Standards, Washington, DC, 1970).

[32] A. Pietzsch, F. Hennies, P. S. Miedema, B. Kennedy, J. Schlappa, T. Schmitt, V. N. Strocov, and A. Föhlisch, Phys. Rev. Lett. 114, 088302 (2015).

[33] E. Ertan, V. Kimberg, F. Gel'mukhanov, F. Hennies, J.-E. Rubensson, T. Schmitt, V. Strocov, K. Zhou, M. Iannuzzi, A. Föhlisch, M. Odelius, and A. Pietzsch, Phys. Rev. B 95, 144301 (2017). 